\title{
Efficacy of INR Self-Management Led by Patients in Prevention of Readmission and Thromboembolic Events on Warfarin or Oral Anticoagulant Therapy
}

\author{
Sana Noor ${ }^{*}$, Sana Sehar ${ }^{2}$, Muhammad Afzal ${ }^{3}$, Dr. Syed Amir Gilani ${ }^{4}$ \\ ${ }^{1}$ BSN(Student),Lahore School of Nursing, The university of Lahore 1-Km Raiwind Road ,Sultan Town, Lahore, Punjab, Pakistan \\ ${ }^{2}$ Assistant professor at LSN(UOL), Lahore School of Nursing, The university of Lahore 1-Km Raiwind Road ,Sultan Town, Lahore, Punjab, Pakistan \\ ${ }^{3}$ Professor at LSN(UOL), Lahore School of Nursing, The university of Lahore 1-Km Raiwind Road, Sultan Town, Lahore, Punjab, Pakistan \\ ${ }^{4}$ Dean Faculty of Allied Health Sciences, Lahore School of Nursing, The university of Lahore 1- Km Raiwind Road, Sultan Town, Lahore, Punjab, \\ Pakistan
}

DOI: $10.36348 /$ sjnhc.2019.v02i12.006

| Received: 05.12.2019 | Accepted: 15.12.2019 | Published: 26.12.2019

*Corresponding author: Sana Noor

\section{Abstract}

Valvular heart diseases affects millions of patients worldwide, and the burden is rising as the population ages. Severe valvular heart disease is associated with significant morbidity and mortality, claiming over 50,000 lives annually in the USA [1]. Coumadin (generic name: warfarin) is an anticoagulant, which stops the formation of blood clots and makes blood thinner (less viscous). As for Vitamin K, it helps the process of blood clotting. In other words, warfarin has the opposite effect to Vitamin K. Warfarin is a widely used and cheap drug which decreases the risk of thrombosis or embolism in patients with diseases such as atrial fibrillation (AF), venous thrombo-embolism, and mechanical heart valve surgeries. Treatment with warfarin requires close monitoring with regular tests of PT-INR for better outcome of patients [2]. The purpose of the study is to shorten and simplify the process for the testing and prescription of warfarin by introducing point of care analysis (POC). The aim was also to evaluate changes in time and cost related to the new processes. Effective management of warfarin therapy is frequently challenged by its difficult pharmacology and adverse effects. One strategy to ensure safe warfarin use is provision of comprehensive warfarin education to patients regarding self-testing and self-management of accurate dose according to INR value. Point of care INR monitoring has shown beneficial impacts on the risk of thromboembolic events, anticoagulation control, patient satisfaction, and quality of life [3].

Keywords: Efficacy Thromboembolic Valvular thrombo-embolism.

Copyright @ 2019: This is an open-access article distributed under the terms of the Creative Commons Attribution license which permits unrestricted use, distribution, and reproduction in any medium for non-commercial use (NonCommercial, or CC-BY-NC) provided the original author and source are credited.

\section{INTRODUCTION}

Valvular heart diseases are associated with high morbidity and mortality, and its prevalence is rising as the ages of population. Treatment of severe valvular disease has historically required surgical correction, which carries its own risks, but there have been significant advances in valve replacement technologies and techniques. The backbone of anticoagulation regimens has been comprised of aspirin, warfarin and Vitamin K [4].

Different Valve surgeries, like single valve, DVR, Bentall are being done in cardiac hoapitals. In which $90 \%$ replaced Valves are mechanical requiring oral anticoagulants (marevan). Due to the affects and interaction of different drugs with marevan (oral anticoagulant) and all possible sufferings, patients undergo in high or low INR ranges. Mostly people are guided by their consultant or they visit hospital based INR clinics but this is time consuming and costly methods. Large prospective trials have done to determine the best and easiest method for their management [1].

Although alternatives are available, but warfarin is still a primary therapeutic drug for managing thrombo-embolic events until long-term safety data on the new anti-thrombotics become available and treatment cost is decreased. While the warfarin have small therapeutic index, complex pharmacology and adverse complications. Therefore, provision of education for patients on warfarin is significant in different clinical settings. With over $50 \%$ of patients are receiving little or no warfarin education from health 
care physician, nurse and pharmacists during admission and after discharge [3].

The formation of a clot in the body is a complex process that involves multiple substances called clotting factors. Warfarin decreases the body's ability to form blood clots by blocking the formation of vitamin $\mathrm{K}$-dependent clotting factors. Vitamin $\mathrm{K}$ is needed to make clotting factors and prevent bleeding. Therefore, by giving a medication that blocks the clotting factors, body can stop harmful clots formation and prevent prevention [4].

In Cardiac surgery (post-operative cases), the use of Long-term oral anticoagulation therapy vitamin $\mathrm{K}$ antagonist of warfarin could be risk of hemorrhagic or thromboembolic events. These life-threatening complications potentially depend on the accuracy of dose adjustment. Therefore, periodically laboratory testing of international normalized ratio (INR) and its dose adjustment are very necessary. Long-term use of oral anticoagulation drugs is a public health problem as, for example, $1 \%$ of the French people use vitamin $\mathrm{K}$ and warfin therapy. During 2007, it requires repeated readmissions because of its iatrogenic effects in France [5].

Home testing devices as point-of-care can be used to measure INR which will improve the compliance, reliability and effectiveness of patients and their families. The therapeutic range of INR can improve patient's safety and quality of life [6].

\section{DISCUSSION}

The Australian National Inpatient Medication Chart (NIMC) highlights the importance of warfarin education by proper documentation. So, this study aimed to review the provision of warfarin education to patients on warfarin and to describe patient characteristics. Some of them do not followed the instructions regarding documentation and under gone high risk redo surgeries. Older patients over 80 years aged are at higher risk as compared to those between 60 to 70 years age. It reported $2 \%$ increased risk of hemorrhagic events per 1 year as age increases. In the present study, more than half of the patients were over 75 years of age and according to the evidence are at an increased risk of warfarin-related adverse events [7].

Bauman, Black et al. [8] have studied 28 children on oral warfarin therapy used to perform selftesting or self-management for about 3 months at home. Time in therapeutic range was $77 \%$ for self-testing and $83 \%$ for self-management. INR greater than 5 was not related to warfarin dose but to unexpected diseases. 90\% Patients were following guidelines with selfmanagement dose adjustment. It showed $50 \%$ reduction in redo surgeries and thromboembolic complications as compared to other people [9].
All health systems do not support specialized oral anticoagulation clinics for optimal management of warfarin for effective patient outcomes. The quality of warfarin management was judged by improving the time in therapeutic range of INR. And it was achieved by good control of INR to about $70 \%$. This protocol was used on 2000 patients and 39 clinics to achieve best results. Clinical nurse, Physician and pharmacist participated effectively in this warfarin management program [10].

Initially Point-of-care INR devices were used to monitor oral anticoagulation and then it extended to the operation theater, emergency rooms, and for patient self-testing at home. To evaluate its performance its results was compared with different laboratories. Its reliability has increased for patients care on chronic oral warfarin therapy. It suggested to health-care physician and nurses to adjust warfarin doses, decision-making and face-to-face education with the client and family [11].

Point-of-care tests (POCT) are performed for patient care. There are many tests as activated clotting time, thrombo-elastography, platelet function and Ddimer etc, are available but the measurement of international normalized ratio (INR) for warfarin dose adjustment is the main test of this kingdom. Warfarin is being used most commonly as oral anticoagulant throughout the world and monitored through INR. It is a mathematical calculation, based on prothrombin time. The therapeutic index of warfarin is narrow as (INR of 2-3) to control coagulation or bleeding, due to complex network of pharmacokinetics and pharmacodynamics. So, INR can be test frequently and easily at home instead of visiting to the laboratory. Moreover it is very beneficial to prevent problems related to venipuncture [11].

Another study showed that the 183 patients that were testing their INR at that time and one year earlier, time in therapeutic range (TTR) was not statistically different, from $75 \%$ to $81 \%$ with the point of care analysis, although margin of error was present in different instruments. The introduction of POC analysis appeared to be safe and cheap that reduces adverse events. And time in therapeutic range was similar among 183 patients with previous test results [12].

A study conducted in Sweden, more than 250 000 patients (approximately $2.5 \%$ of the population) are under going on warfarin therapy. Many patients are treated with high cost new oral anticoagulants (NOAC) instead of warfarin. But the number of patients with warfarin is still high and need to be managed with the best possible practice [12].

A study with POC was performed during six months in 2014. Time expenditure, cost, time in therapeutic range TTR, and adverse events related to 
warfarin treatment were recorded. It showed $60 \%$ decline in serious bleeding events in valvular patients who had received warfarin education from the health care practitioners as doctors, nurses and pharmacists [3].

In this study data was collected from 96 patients from two different hospital settings. There was no significant difference in the characteristics of patients between the two hospitals, apart from their warfarin taking history. The most prevalent indication for warfarin was atrial fibrillation, followed by deep vein thrombosis, heart valve replacement, pulmonary embolism and embolic stroke. Interview was taken from patients after provision of warfarin education, 68 (71\%) were available to answer the questions out of the 96 patients. who showed better results with documentation during home stay [13].

\section{Problem Focused}

The ratio of people is increasing day by day, approximately $1.8 \%$ of people are having oral anticoagulant therapy. Prothrombin time and international normalized ratio (PT/INR) values are considered in therapeutic range from 2-3. But in case of valve surgery its management is quiet difficult. Any deviation of INR from normal range may risk for bleeding or thromboembolic events. Uncontrolled blood pressure, positive family history of cardiac diseases, history of stroke and gastric ulcer can increase the risk of bleeding in such patients. It will increase the high risk readmission, redo valve surgeries, due to struck valve, atrial fibrillation and infective endocarditis and will increase the morbidity rate. Strict monitoring is needed on daily basis to adjust accurate dose of Warfarin post-operatively and later on once a week [13].

Many reasons can explain this problem: the lack of education on discharge, multiple medications, inadequate dietary regime, repeated visit to laboratory, interruption in jobs, and increased absenteeism from school and colleges. Patients used to visit different laboratories to give their blood samples and wait for many hours for results. Which is very time consuming process for the patients who are on warfarin therapy [1].

Many patients with several comorbidities were also taking omeprazole, fluconazole (Diflucan) Aspirin or aspirin-containing products as Ibuprofen (Advil, Motrin IB, others) or naproxen sodium, Acetaminophen (Tylenol, others) medicines, which showed significant interactions with warfarin. Medications that treat abnormal heart rhythms, such as amiodarone (Amiodarone $\mathrm{HCl}$, Pacerone) Antacids or laxatives etc. Provision of education is necessary for such group of people [2].

\section{Management process}

- On the day of surgery, with low risk of bleeding Recommend warfarin at the patient's usual dose and therapeutic anticoagulant until INR is in therapeutic range is achieved. No anticoagulation therapy is needed for first 24 hours [14].

- While on the $1^{\text {st }}$ day, continue warfarin, as dosing guidelines above OR - IV heparin infusion according to daily based results of INR value.

- Monitor INR every 2-3 day within range (2.5 to 3.5 ), and continue therapeutic dose or IV heparin infusion until INR is in the target range on 2 consecutive days.

- Then fix the warfarin dose for one week on discharge, and educate the patient how to manage its dose at home.

- Provide education about dietary regime, not to use green leafy vegetables which will derange the clotting time, hence the complication will arise.

- Encourage for laboratory test of INR at least once a week to document the results and adjust warfarin dose accordingly.

\section{Problem Solving Process}

\section{Define the problem}

Management of Warfarin doses according to INR values in valve surgeries after discharge.

\section{Gather information}

Information was collected from different patients in public hospitals on readmission with deranged INR values.

\section{Analyze the information}

Analysis of data was done by comparing to other patients who was using self-testing and selfmanagement strategies.

\section{Develop Solution}

Ensure provision of education related to selftesting and management of accurate doses by point of care analysis.

\section{Make a decision}

Decision was taken to enhance the knowledge of patients and families by introducing self-testing and management methods for better outcome of patients and quality of life.

\section{Implementation of decision}

Structured education, self-management strategies and knowledge by trained health care professionals is made accessible for children, adults and family to reduce the bleeding and thromboembolic events and the goal for adjustment of dose by frequent testing of INR was achieved accurately. 


\section{Evaluation of the solution}

Implementation of solution done actively which reduced the number of readmissions on warfarin therapy

\section{CONCLUSION}

The high risk for reoperation of young patients implanted with bio-prosthetic valve (BV) or mechanical valve (MV), BV and the availability of a safer and easier way to conduct long-term vitamin $\mathrm{K}$ antagonism treatment, such as the use of point-of-care devices, should be considered when the type of valve must be chosen. Provision of education and documentation made assessable to patients related to self-testing and self-management process to adjust accurate dose after discharge. The results of this study show that the introduction of Point of care testing decreased the number of steps and parties involved in the warfarin prescription process.

\section{REFERENCES}

1. Poli, D., Antonucci, E., Pengo, V., Grifoni, E., Maggini, N., Testa, S., \& Paparo, C. (2018). Risk of reoperation in bioprosthetic valve patients with indication for long-term anticoagulation. Results from the observational retrospective multicentre PLECTRUM study. Open heart, 5(2), e000837.

2. Morgan, T. J., \& Anstey, C. M. (2019). Expanding the boundaries of point of care testing. Journal of clinical monitoring and computing, 1-3.

3. Sharma, P., Scotland, G., Cruickshank, M., Tassie, E., Fraser, C., Burton, C., \& Brazzelli, M. (2015). Is self-monitoring an effective option for people receiving long-term vitamin $\mathrm{K}$ antagonist therapy? A systematic review and economic evaluation. BMJ open, 5(6), e007758.

4. Cigarroa, R., \& Elmariah, S. (2018). Anticoagulation Management After Transcatheter and Surgical Valve Replacement. Current treatment options in cardiovascular medicine, 20(5), 42.

5. Pozzi, M., Mitchell, J., Henaine, A. M., Hanna, N., Safi, O., \& Henaine, R. (2016). International normalized ratio self-testing and self-management: improving patient outcomes. Vascular health and risk management, 12, 387.

6. Bonar, R. (2015). International normalized ratio monitoring of vitamin $\mathrm{K}$ antagonist therapy: comparative performance of point-of-care and laboratory-derived testing. Seminars in thrombosis and hemostasis, Thieme Medical Publishers.

7. Nasser, S. (2012). "Documentation of warfarin education provided to hospital patients: a clinical audit." 42(2): 129-133.

8. Bauman, M. E., Black, K., Bauman, M. L., Bruce, A. A. K., Kuhle, S., Bajzar, L., \& Massicotte, M. P. (2010). EMPoWarMENT: Edmonton pediatric warfarin self-management pilot study in children with primarily cardiac disease. Thrombosis research, 126(2), e110-e115.

9. Liu, W. (2018). "Anticoagulation management by physician-clinical pharmacist team for patients with valvular atrial fibrillation." 17(12): 997-1001.

10. Rose, A. E. (2017). "Improving warfarin management within the medical home: a healthsystem approach." 130(3): 365. e367-365. e312.

11. Moiz, B. (2018). "Prospective comparison of pointof-care device and standard analyzer for monitoring of international normalized ratio in outpatient oral anticoagulant clinic." 24(7): 1153-1158.

12. Fernholm, R., \& J. J. B. O. Q. Hermansson. (2015). "Introduction of point of care analysis for prescribing warfarin at a Swedish primary care centre." 4(1): u208905. w203692.

13. Karlsson, T. J. B. O. Q. (2016). "A project to improve the management of patients on warfarin in a primary care setting through the introduction of a POC analysis." 5(1): u211003. w214421.

14. Brew, S., \& S. J. A. M. P. J. A.-D. Bolsin. (2018). "Management of Elective Surgery Patients on Long Term Warfarin Bridged with Enoxaparin." 10.

15. Morgan, C. L. (2009). "Warfarin treatment in patients with atrial fibrillation: observing outcomes associated with varying levels of INR control." 124(1): 37-41. 